\section{D) Check for updates}

Cite this: Dalton Trans., 2020, 49 9306

Received 1st May 2020,

Accepted 17th June 2020

DOI: $10.1039 / \mathrm{d} 0 \mathrm{dt} 01587 \mathrm{c}$

rsc.li/dalton

\title{
Correlations of acidity-basicity of solvent treated layered double hydroxides/oxides and their $\mathrm{CO}_{2}$ capture performance $\uparrow$
}

\begin{abstract}
D. W. Justin Leung, Chunping Chen, Jean-Charles Buffet (D) and Dermot O'Hare (D) *
The basicity and acidity of solvent-treated layered double hydroxide (ST-LDHs) and their layered double oxides (ST-LDOS) have been fully studied using Hammett titration, in situ FTIR, $\mathrm{CO}_{2}$-TPD and $\mathrm{NH}_{3}$-TPD. Five solvents (ethanol, acetone, isopropanol, ethyl acetate and 1-hexanol) were selected to treat $\left[\mathrm{Mg}_{0.72} \mathrm{Al}_{0.28}(\mathrm{OH})_{2}\right]\left(\mathrm{CO}_{3}\right)_{0.14}\left(\mathrm{Mg}_{2.5} \mathrm{Al}-\mathrm{CO}_{3} \mathrm{LDH}\right)$ and compared with traditional LDH co-precipitated from water. The Brønsted basicity strength of the ST-LDHs and ST-LDOs increased but was accompanied by a decrease in basic site density. In addition, the Lewis acidity of ST-LDOs also changes significantly, with medium strength Lewis acid sites dissapearing after solvent treatment. We found that the $\mathrm{CO}_{2}$ capture capacity of solvent treated LDOs is $50 \%$ higher than that of traditional co-precipitated LDO sample. The ethanol treated LDO exhibited the highest $\mathrm{CO}_{2}$ uptake of $1.01 \mathrm{mmol} \mathrm{g}^{-1}$. The observed $\mathrm{CO}_{2}$ capture performance of the ST-LDOs correlates linearly with the ratio of total acid sites to total basic sites.
\end{abstract}

\section{Introduction}

Layered double hydroxides (LDHs), also known as anionic clays, are a family of inorganic layered materials with a general composition $\left[\left(\mathrm{M}_{1-x}^{z+} \mathrm{M}_{x}^{\prime}{ }^{y+}(\mathrm{OH})_{2}\right)\right]^{w+}\left(\mathrm{A}^{n-}\right)_{w / n} \cdot m \mathrm{H}_{2} \mathrm{O}$, where $\mathrm{M}^{z+}$ and $\mathrm{M}^{\prime{ }^{+}}$are one or more different metal cations, $z$ can be 1 or 2 and $y$ can be 3 or 4 ; overall the metal hydroxide layer charge $(w)$ is determined by $w=z(1-x)+x y-2$ which is compensated by $w / n \mathrm{~A}^{n-}$ interlayer anions. ${ }^{1}$ Most commonly, LDHs contain both $\mathrm{M}^{2+}$ and $\mathrm{M}^{3+}$ cations, and $x$ has been found to give crystalline phase pure LDHs when $0.35 \geq x \geq 0.16{ }^{2}$ Water molecules may also be incorporated into the interlayer region during synthesis, which form hydrogen bonds to the hydroxyl groups on the surface of the cationic layers as well as solvating the hydrophilic interlayer anions. This stabilises the layered structure while still maintaining anion mobility. ${ }^{3}$ Upon thermal treatment at low temperatures $\left(100-200{ }^{\circ} \mathrm{C}\right)$, LDHs lose both surface-bound and interlamellar water. At $300-600{ }^{\circ} \mathrm{C}$, LDOs are formed by de-hydroxylation and aniondecomposition. ${ }^{4-7}$ At higher temperatures, LDHs irreversibly decompose to form crystalline spinel phases that cannot be reconstructed back. ${ }^{8}$

Chemistry Research Laboratory, Department of Chemistry, University of Oxford, 12 Mansfield Road, Oxford, OX1 3TA, UK. E-mail: dermot.ohare@chem.ox.ac.uk; Tel: $+44(0) 186527268$

$\dagger$ Electronic supplementary information (ESI) available: Characterisation technique details, XRD patterns and $\mathrm{CO}_{2}$ adsorption curves. See DOI: 10.1039/ d0dt01587c
Due to their inherent basicity, both LDHs and LDOs have been widely used as heterogeneous catalysts. ${ }^{9-11}$ Efforts have been made to increase their basicity in order to amplify their catalytic behaviour by doping with various cations into the layer structure or by surface impregnation. ${ }^{12,13}$ They have also been attracting increasing interest for $\mathrm{CO}_{2}$ capture, many studies have been published on the effects of calcination temperatures, cation doping and intercalated anion on $\mathrm{CO}_{2}$ capture performance. ${ }^{14-19}$ However, due to the hydrophilic nature of LDHs, the primary platelet particles are usually highly aggregated by a strong hydrogen bonding network, resulting in low surface areas and formation of large platelet agglomerates, which can severely limit their use in catalysis and $\mathrm{CO}_{2}$ capture. ${ }^{20}$ O'Hare and co-workers have developed post synthesis solvent treatment (ST) methods to create highly dispersed LDHs (ST-LDHs). Initially, an aqueous miscible organic solvent treatment method (AMOST) was reported; ${ }^{21}$ more recently, an aqueous immiscible organic (AIM) solvent treatment has proved to be equally effective. ${ }^{22}$ It has been proposed that the solvent penetrates the interlayer region to varying extents, disrupting the hydrogen bonding network and displacing any surface bound water. This weakens the interlayer interactions, allowing the cationic layers to be delaminated, resulting in a highly dispersed platelets with high surface area and large pore volume. ${ }^{22,23}$ These newly discovered LDHs families have been found to be highly effective solid supports for single-site catalysts in slurry phase ethylene polymerisation. ${ }^{24,25}$ Very recent studies have been carried out on the impact of AMO-LDHs (using acetone as AMO solvent) 
on $\mathrm{CO}_{2}$ capture performance. ${ }^{26,27}$ However, there has been no systematic study of the changes in surface chemistry of LDHs following solvent treatment and in particular how this may relate to their use as $\mathrm{CO}_{2}$ adsorbents. Understanding the changes in surface chemistry of solvent treated LDHs will allow for optimisation of their catalytic and absorbent abilities.

Herein, we have investigated the acid and base properties of solvent treated LDHs (AMO- and AIM-LDHs) and their respective LDOs. The Brønsted basicity of both solvent treated LDHs and LDOs were evaluated using Hammett indicators in solution. The Lewis basicity and acidity of solvent treated LDOs were analysed using in situ FTIR and temperature programmed desorption (TPD). The $\mathrm{CO}_{2}$ capture performance of the ST-LDHs was evaluated using chemisorption experiments.

\section{Results and discussion}

\section{Basicity studies of ST-LDHs}

A conventional $\left[\mathrm{Mg}_{0.72} \mathrm{Al}_{0.28}(\mathrm{OH})_{2}\right]\left(\mathrm{CO}_{3}\right)_{0.14}\left(\mathrm{Mg}_{2.5} \mathrm{Al}-\mathrm{CO}_{3}\right) \mathrm{LDH}$ (LDH-W) was synthesised using an adapted literature co-precipitation procedure in water. ${ }^{21}$ Solvent treated LDH samples (ST-LDHs) were prepared by re-dispersing a $30 \%$ solid content aqueous dispersion of $\mathrm{Mg}_{2.5} \mathrm{Al} \mathrm{CO} \mathrm{CDH}_{3} \mathrm{Let}$ cake in the appropriate dispersing solvent for $4 \mathrm{~h}$. The dispersing solvents used in this study were ethanol, acetone, isopropyl alcohol, ethyl acetate and 1-hexanol to yield LDH-E, LDH-A, LDH-IPA, LDH-EA and LDH-1H, respectively. The chemical composition of all the LDHs are provided in Table $\mathrm{S} 1 . \dagger$

The powder X-ray diffraction (XRD) data for all the samples (Fig. $\mathrm{S} 1 \dagger$ ) are typical diffractions of an $\mathrm{Mg}_{2.5} \mathrm{Al}-\mathrm{CO}_{3} \mathrm{LDH}$. As previously reported, we observe small variations in the lattice constants (Table S2 $\dagger$ ) and the peak widths of the Bragg reflections for ST-LDHs compared to the conventional sample (LDH-W). ${ }^{21,22}$ There is a large increase in the available surface area and pore volume of the ST-LDHs compared to LDH-W (Table S3†). LDH-E showed the highest surface area of $196 \mathrm{~m}^{2}$ $\mathrm{g}^{-1}$ while LDH-EA had the highest pore volume of $0.83 \mathrm{~cm}^{3}$ $\mathrm{g}^{-1}$.

Variation of Brønsted basicity of these materials was studied using Hammett indicators, three indicators were used: bromothymol blue $\left(\mathrm{p} K_{\mathrm{a}}=7.1\right)$, phenolphthalein $\left(\mathrm{p} K_{\mathrm{a}}=9.3\right)$ and clayton yellow $\left(\mathrm{p} K_{\mathrm{a}}=12.7\right)$. For the purpose of this study, Brønsted basic sites $\mathrm{pH}>12.7,9.3 \leq \mathrm{pH} \leq 12.7$ and $7.1 \leq \mathrm{pH}$ $\leq 9.3$ are assigned to the label 'strong', 'medium' and 'weak', respectively. All samples showed similar responses of having fewer medium Brønsted basic sites compared to the strong and weak ones (Fig. 1). LDH-IPA exhibited the highest number of medium basic sites $\left(0.15 \mathrm{mmol} \mathrm{g}^{-1}\right)$, double that of any other ST-LDHs. Analysis of the total number of Brønsted basic sites (Table 1), revealed that all ST-LDHs exhibited a higher total number compared to LDH-W $\left(0.41 \mathrm{mmol} \mathrm{g}^{-1}\right)$. LDH-IPA and LDH-1H showed the highest total basicity at $0.67 \mathrm{mmol}$ $\mathrm{g}^{-1}$. However, once the surface area was taken into account, the AMO-LDHs showed lower basic site densities compared to

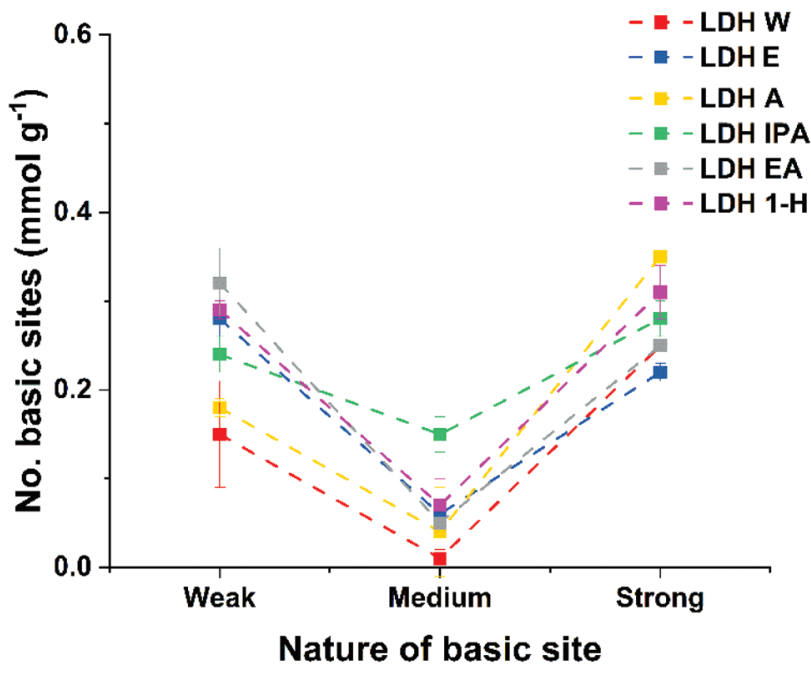

Fig. 1 Plot of the total number of basic sites for ST-LDHs (dotted lines are just a guide to the eye).

Table 1 Summary of titration data of pre-treated LDH samples

\begin{tabular}{lll}
\hline Sample & Total basic sites $\left(\mathrm{mmol} \mathrm{g}^{-1}\right)$ & Basic site density $\left(\mu \mathrm{mol} \mathrm{m}{ }^{-2}\right)$ \\
\hline LDH-W & $0.41 \pm 0.06$ & $4.52 \pm 0.65$ \\
LDH-E & $0.57 \pm 0.02$ & $2.92 \pm 0.10$ \\
LDH-A & $0.57 \pm 0.01$ & $3.29 \pm 0.06$ \\
LDH-IPA & $0.67 \pm 0.02$ & $3.62 \pm 0.01$ \\
LDH-EA & $0.61 \pm 0.04$ & $3.48 \pm 0.23$ \\
LDH-1H & $0.67 \pm 0.01$ & $4.04 \pm 0.06$
\end{tabular}

LDH-W (4.52 $\mu \mathrm{mol} \mathrm{m} \mathrm{m}^{-2}$ ). LDH-1H had the highest Brønsted basic site density of all ST-LDHs at $4.04 \mu \mathrm{mol} \mathrm{m}^{-2}$.

The solvent treatment method increases LDH surface area without changing the structural arrangement of the cation within the layers. Although solvent treatment increases the available specific surface area, a priori, it would not be expected to change the basic site density of all samples. Especially as the previous reports suggest that the pre-treatment temperature of $200{ }^{\circ} \mathrm{C}$ only removes solvents leaving the underlying $\mathrm{LDH}$ structure intact. ${ }^{21,28,29}$ The decrease in the total basic site density for some solvent treated samples may be due to these solvents blocking basic sites. This site blocking could be a result of trace amounts of solvent absorbed on the surface. Perhaps treatment temperature of $200{ }^{\circ} \mathrm{C}$ not sufficiently high to desorb all surface sorbed solvents. It has been shown previously that hydroxyl-containing molecules may bind to the surface of an $\mathrm{LDH}$, resulting in the surface hydroxyl groups not being accessible. ${ }^{30}$

In situ Fourier-transform infra-red (FTIR) spectroscopy was used to probe the LDHs' Lewis basicity. As shown in Fig. S2, $\dagger$ LDH-W exhibits a strong absorbance between 1360-1400 $\mathrm{cm}^{-1}$ and a less intense absorbance between 1470-1580 $\mathrm{cm}^{-1}$, which are associated with strong Lewis basic sites due to monodentate carbonate surface coordination. A weak bidentate carbonate stretching vibration $\left(1580-1670 \mathrm{~cm}^{-1}\right)$ can be 
assigned to a medium strength basic site in LDH-W. These findings are consistent with previously reported literature results. ${ }^{31,32}$ Solvent treated $\mathrm{LDH}$ samples exhibit varying levels of both strong and medium strength Lewis basic sites. Solvent treatment appears to alter the overall Lewis basicity profile of ST-LDHs. In particular, both LDH-E and LDH-1H exhibited enhanced numbers of medium strength basic sites, while the medium basic sites are eliminated by treatment with acetone and isopropanol.

\section{Basicity and acidity studies of ST-LDOs}

Solvent treated LDOs (ST-LDOs) were prepared by calcining the appropriate $\mathrm{ST}-\mathrm{LDH}$ at $450{ }^{\circ} \mathrm{C}$ for $12 \mathrm{~h}$ with a heating ramp rate of $5{ }^{\circ} \mathrm{C} \mathrm{min}^{-1}$. The LDO products were named LDO-W, LDO-E, LDO-A, LDO-IPA, LDO-EA and LDO-1H by calcination of the appropriate ST-LDH respectively. As shown in Fig. 2, the general trend for ST-LDO samples (except LDO-EA) is that the number and strength of the Brønsted sites increases compared to the parent LDH. This increase in the number of strong Brønsted basic sites is due to the decomposition of hydroxide groups to unsaturated oxygen atoms and metal-oxygen pairs on the surface of the LDH nanosheets, which have higher basicity. Interestingly, both LDO-E and LDO-IPA exhibit a negligibly low number of weak basic sites and LDO-EA did not have any medium sites. LDO-1H showed the highest number of total Brønsted basic sites at $1.58 \mathrm{mmol} \mathrm{g}^{-1}$. In general, both the total number of basic sites and the basic site density of LDOs increases from that of their parent $\mathrm{LDH}$. This is due to an increase in surface area as the LDH nanosheets start decomposing by dehydroxylation, decomposition of intercalated carbonate, and loss of interlayer water. Furthermore, some aluminium ions migrate from octahedral into tetrahedral sites ${ }^{33}$ (leading to exposure of more basic sites). Solvent treated LDOs (except LDO-1H) displayed a lower basic site density compared to LDO-W $\left(4.83 \mu \mathrm{mol} \mathrm{m}{ }^{-2}\right)$, while LDO-1H

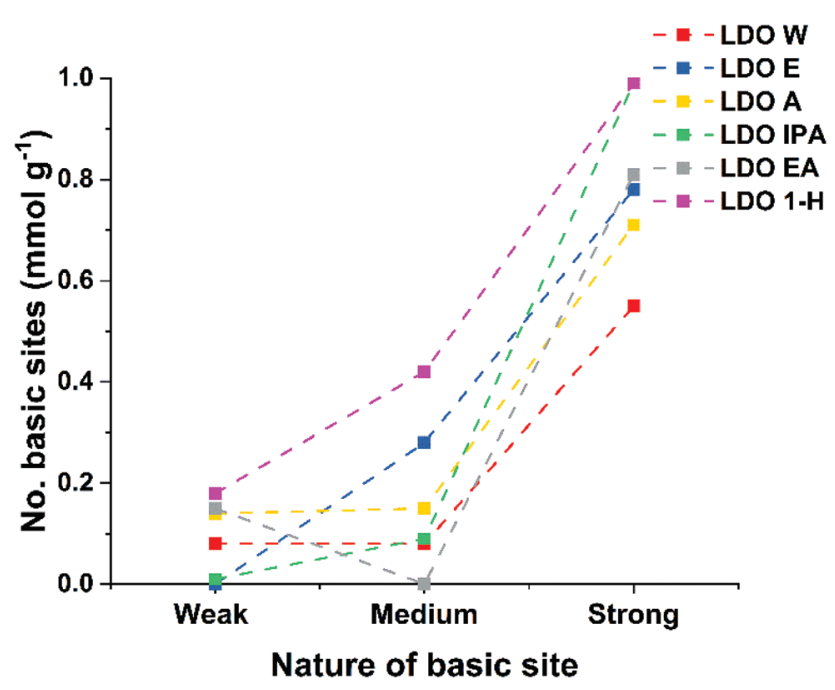

Fig. 2 Titration plots of all basic sites of various ST-LDOs (dotted lines are just a guide to the eye).
Table 2 Summary of BET surface area and titration data of LDO samples

\begin{tabular}{llll}
\hline Sample & $\begin{array}{l}\text { Surface area } \\
\left(\mathrm{g} \mathrm{m}^{-2}\right)\end{array}$ & $\begin{array}{l}\text { Total basic sites } \\
\left(\mathrm{mmol} \mathrm{g}^{-1}\right)\end{array}$ & $\begin{array}{l}\text { Basic site density } \\
\left(\mu \mathrm{mol} \mathrm{m}^{-2}\right)\end{array}$ \\
\hline LDO-W & 147 & 0.71 & 4.83 \\
LDO-E & 310 & 1.04 & 3.35 \\
LDO-A & 251 & 1.00 & 3.99 \\
LDO-IPA & 286 & 1.09 & 3.81 \\
LDO-EA & 243 & 0.89 & 3.67 \\
LDO-1H & 273 & 1.58 & 5.79
\end{tabular}

has the highest total number of basic sites and highest basic

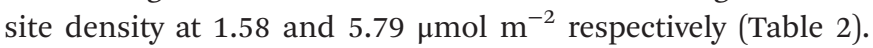
Both $\mathrm{CO}_{2}$ and $\mathrm{NH}_{3}$ temperature programmed desorption (TPD) have been used to probe the Lewis basicity and acidity for the LDO samples.

There is no noticeable difference in the general desorption profiles for the $\mathrm{CO}_{2}$ TPD (Fig. 3a), indicating that there is no
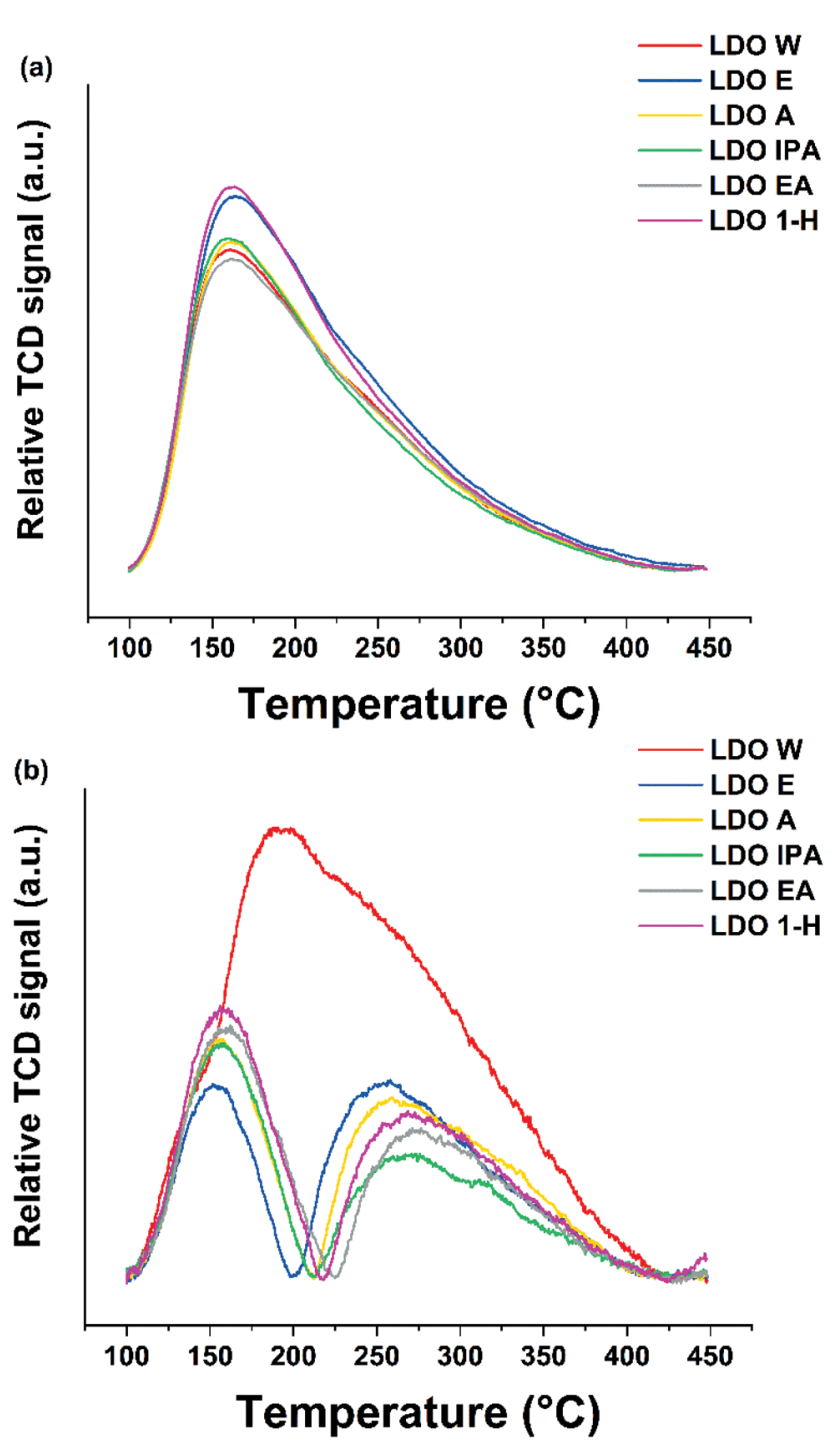

Fig. 3 (a) $\mathrm{CO}_{2}$ and (b) $\mathrm{NH}_{3}$ TPD profiles of ST-LDO samples. 
change in the distribution profile of Lewis basic sites. The samples those treated with acetone, IPA and ethyl acetate had very similar number of basic sites $c a$. $\left(1.96 \mathrm{mmol} \mathrm{g}^{-1}\right)$. LDO-E and LDO-1H had Lewis basic sites in excess of $2.2 \mathrm{mmol} \mathrm{g}^{-1}$.

However, once the various surface areas of the LDOs are taken into account, all solvent treated samples present similar Lewis basic site density in the range of 7.22-8.10 $\mu \mathrm{mol} \mathrm{m} \mathrm{m}^{-2}$ (Table 3), which are much lower than that of LDO-W $\left(13.29 \mu \mathrm{mol} \mathrm{m}{ }^{-2}\right)$. The $\mathrm{NH}_{3}$ TPD experiments probe the Lewis acidity (Fig. 3b). LDO-W exhibited an intense, wide peak over the range of $100-450{ }^{\circ} \mathrm{C}$. In contrast, ST-LDOs only exhibited two distinct individual peaks in the low temperature range (100-200 ${ }^{\circ} \mathrm{C}$ ) and high temperature range (200-450 $\left.{ }^{\circ} \mathrm{C}\right)$, which are assigned to the hydrogen bonding between $\mathrm{NH}_{3}$ and surface oxide/hydroxide groups and the strong strengths of Lewis acid sites, respectively. The medium strength Lewis acid sites disappeared after solvent treatment and their overall intensity decreased the compared to LDO-W. We believe this may arise because solvents strongly interact with acid sites during treatment, leading to blocking of some of the acid sites even after calcination. The total acidity of LDO-W is $0.38 \mathrm{mmol}$ $\mathrm{g}^{-1}$ while the solvent treated LDOs had a significantly lower value in the range of $0.16-0.21 \mathrm{mmol} \mathrm{g}^{-1}$, where LDO-IPA has the lowest acidity value of $0.16 \mathrm{mmol} \mathrm{g}^{-1}$. Considering the Lewis acidic site density, all solvent treated LDO samples exhibit a Lewis acidic site density 3-5 times lower than that of LDO-W. It is interesting to find that the Lewis acid/base ratio of LDOs can be easily tuned from 0.08 to 0.19 by using different solvents. The Lewis acid/base ratio shows a correlation with the surface areas of the LDOs: the higher the surface area, the lower the ratio (Fig. S3†). This is due to both surface area and reduction in acid sites being a consequence of solvent penetration. A high surface area is the result of effective solvent replacement of surface and interlayer water molecules. This results in more solvent molecules being incorporated into the structure which will block more acid sites upon heat treatment.

Prinetto et al. have shown that the peak in the $\mathrm{NH}_{3} \mathrm{TPD}$ at $150{ }^{\circ} \mathrm{C}$ is due to the hydrogen bonding between $\mathrm{NH}_{3}$ and surface oxide/hydroxide groups (Scheme $\mathrm{S} 1(\mathrm{a}) \dagger$ ). ${ }^{34}$ The resulting $\mathrm{NH}_{3}$ TPD features result from a combination of two different bonding modes involving the bonding between the nitrogen and the surface, and hydrogen bonding of an ammonia hydrogen to a neighbouring oxygen site (Scheme S1(b)†). These two bonding modes could not be resolved into separate features. However, it is important to note that both modes involve bonding to metal sites and both the medium and strong sites see a complete suppression and reduction in intensity respectively. While the $\mathrm{NH}_{3}$ bonding modes mainly occur through the metal sites, the $\mathrm{CO}_{2}$ bonding modes mainly concern surface oxygen sites. This indicates that the oxygen sites on the surface treated samples seem unaffected (Fig. 3a).

The change in the $\mathrm{NH}_{3}$ TPD curves, along with the reduction in basic site densities suggests that blocking of specific surface metal sites occurs upon solvent treatment. Our previous work has shown that the dispersing solvents remain on the surface of the AMO/AIM-LDH to variable degrees and these may result in the blocking of some active sites resulting in a decrease in basic and acidic site densities.

\section{$\mathrm{CO}_{2}$ adsorption on LDOs}

The $\mathrm{CO}_{2}$ adsorption capacity of the various ST-LDOs at $40{ }^{\circ} \mathrm{C}$ is shown in Fig. 4 and $\mathrm{S} 4-\mathrm{S} 9 . \dagger \mathrm{CO}_{2}$ adsorption by LDOs is a process involving both chemisorption and physisorption. For each sample, we observe that $\mathrm{CO}_{2}$ physisorption is around twice that of chemisorption. LDO-W showed a total $\mathrm{CO}_{2}$ adsorption of $0.64 \mathrm{mmol} \mathrm{g}^{-1}\left(0.44\right.$ and $0.20 \mathrm{mmol} \mathrm{g}^{-1}$ for phy-

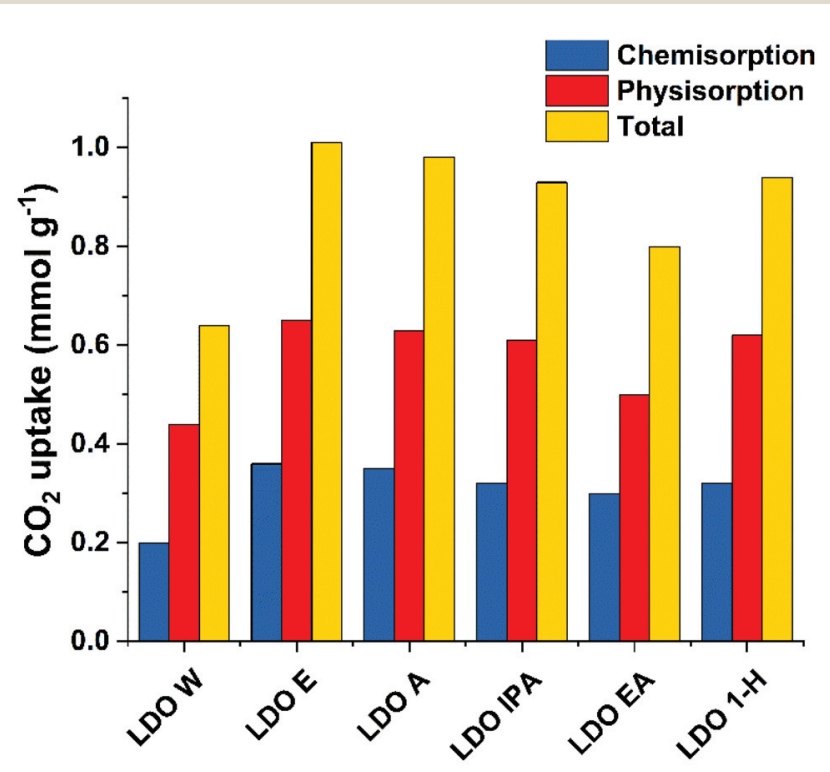

Fig. $4 \mathrm{CO}_{2}$ capture of various ST-LDO samples at $40{ }^{\circ} \mathrm{C}$ and $100 \mathrm{kPa}$.

Table 3 Summary of $\mathrm{CO}_{2}$ and $\mathrm{NH}_{3}$ TPD of LDO samples

\begin{tabular}{llllll}
\hline Sample & $\begin{array}{l}\text { Total basic } \\
\text { sites }\left(\mathrm{mmol} \mathrm{g}^{-1}\right)\end{array}$ & $\begin{array}{l}\text { Basic site } \\
\text { density }\left(\mu \mathrm{mol} \mathrm{m}^{-2}\right)\end{array}$ & $\begin{array}{l}\text { Total acidic } \\
\text { sites }\left(\mathrm{mmol} \mathrm{g}^{-1}\right)\end{array}$ & $\begin{array}{l}\text { Acidic site } \\
\text { density }\left(\mu \mathrm{mol} \mathrm{m}^{-2}\right)\end{array}$ & $\begin{array}{l}\text { Total acidic sites/total } \\
\text { basic sites }\end{array}$ \\
\hline LDO-W & 1.95 & 13.29 & 0.38 & 2.59 & 0.19 \\
LDO-E & 2.24 & 7.22 & 0.19 & 0.61 & 0.08 \\
LDO-A & 1.97 & 7.86 & 0.21 & 0.84 & 0.11 \\
LDO-IPA & 1.97 & 6.88 & 0.16 & 0.57 & 0.08 \\
LDO-EA & 1.95 & 8.03 & 0.19 & 0.77 & 0.10 \\
LDO-1H & 2.21 & 8.10 & 0.21 & 0.77 & 0.09
\end{tabular}


sisorption and chemisorption respectively). Overall, ST-LDOs show a $\mathrm{CO}_{2}$ adsorption capacity increase of $\mathrm{ca}$. 50\% in both physisorption and chemisorption.

LDO-E exhibited the highest total $\mathrm{CO}_{2}$ uptake of $1.01 \mathrm{mmol}$ $\mathrm{g}^{-1}$, which represents a 58\% increase compared with LDO-W. This is higher than previously reported figures for $\mathrm{CO}_{2}$ capture using LDOs under similar conditions. Sharma et al. have reported the highest to date of $0.38 \mathrm{mmol} \mathrm{g}^{-1}$ at $30{ }^{\circ} \mathrm{C}$ using a calcination temperature of $350{ }^{\circ} \mathrm{C}$ while Shang et al. reported a total $\mathrm{CO}_{2}$ capture value of $0.83 \mathrm{mmol} \mathrm{g}^{-1}$ for an acetonetreated $\mathrm{Mg}_{3} \mathrm{Al} \quad \mathrm{CO}_{3} \mathrm{LDH}$ (pretreatment temperature of $\left.180{ }^{\circ} \mathrm{C}\right) .{ }^{26,35}$ LDO-EA exhibited a lowest $\mathrm{CO}_{2}$ uptake of $0.80 \mathrm{mmol} \mathrm{g}^{-1}$ amongst the ST-LDO samples tested. The chemisorption value of $0.30 \mathrm{mmol} \mathrm{g}^{-1}$ was comparable with the other solvent treated samples, but the physisorption figure has decreased due to the lower surface area.

It can be observed that the $\mathrm{CO}_{2}$ adsorption per $\mathrm{g}$ of all LDOs have a positive correlation with their surface area (Fig. S10†). Simply expressed, the higher the surface area, the more active adsorption sites are exposed, leading to a higher $\mathrm{CO}_{2}$ adsorption.

All solvent treated samples, with the exception of LDO-EA, exhibited a physisorption value similar to the total adsorption value of LDO-W. There seems to be a linear correlation $(R=$ 0.94) relating the $\mathrm{CO}_{2}$ adsorption per surface area to the ratio total acidic to basic sites ( $\mathrm{mmol} \mathrm{g}^{-1}$ ) of the LDOs (Fig. 5). This atomic-level origin of this linear relationship to not clear at this time. However, it is worth noting that the acid sites as measured heavily involve the surface hydroxyl groups as shown in Scheme S1. $\dagger$ These hydroxyl groups can participate in $\mathrm{CO}_{2}$ adsorption via weak $\mathrm{CO}_{2}$ bidentate bonding. But this bonding mode is not present in the $\mathrm{CO}_{2}$ TPD due to the adsorption temperature of $100{ }^{\circ} \mathrm{C} .{ }^{31,34}$ This observation suggests that solvent treatment not only disperses individual nanosheets increasing the accessible surface area but also the solvent

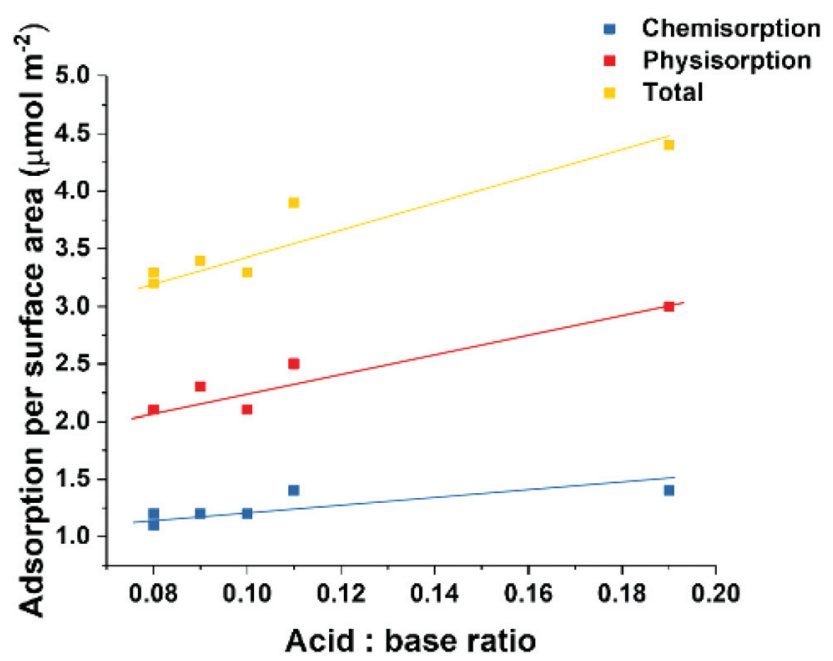

Fig. 5 Molar $\mathrm{CO}_{2}$ adsorption per $\mathrm{m}^{2}$ as a function of the ratio of total acid sites to total basic sites for all ST-LDOs. interacts with the surface and selectively blocks acidic sites, leading to a slightly less active surface for $\mathrm{CO}_{2}$ adsorption.

\section{Conclusions}

A quantitative and comparative study of the basic and acidic features of conventional water washed and solvent treated LDHs and LDOs has been performed. Titration showed that solvent treatment leads to increase in Brønsted basicity in the LDHs while decreasing the basic site density. For Lewis basicity, all LDH samples showed evidence for monodentate carbonate binding while only LDH-E and LDH-1H exhibit stretching vibrations assigned to bidentate carbonate bonding for the solvent treated samples. These results show that changes in the Lewis basicity of LDHs can be achieved using the solvent treatment methods.

Titration experiments on LDOs showed a general increase in total basic sites and basic site density compared to the parent LDH. All ST-LDOs exhibited lower basic site densities compared to LDO-W. All ST-LDOs showed no change in the distribution of basic strengths. The $\mathrm{NH}_{3}$ desorption profiles for ST-LDOs showed an elimination of the medium strength peak along with a reduction in intensity of the strong acidic peak, both of which involve $\mathrm{NH}_{3}$ bonding to the surface metal sites. This evidence, along with the reduction in basic site densities of solvent treated samples, strongly suggests that solvent molecules coordinate to the metal sites upon thermal treatment, which leads to blocking of active sites.

ST-LDOs displayed an increase in $\mathrm{CO}_{2}$ uptake capacity compared to conventional LDO (LDO-W; $0.64 \mathrm{mmol} \mathrm{g}^{-1}$ ). LDO-E exhibited the highest total $\mathrm{CO}_{2}$ uptake of $1.01 \mathrm{mmol} \mathrm{g}^{-1}$, which represents a 58\% increase compared with LDO-W and we believe currently the highest value reported in the literature.

We found that the $\mathrm{CO}_{2}$ adsorption per surface area increases with an increasing acid/base ratio. Overall, study has found that the acid/basic properties of $\mathrm{LDH}$ can be fine-tuned by using (AMO or AIM) solvent treatments, which offers promising opportunities in selective catalysis and adsorption.

\section{Experimental}

\section{Synthesis of ST- $\mathrm{Mg}_{2.5} \mathrm{Al} \mathrm{CO}$-LDHs}

$\left[\mathrm{Mg}_{0.72} \mathrm{Al}_{0.28}(\mathrm{OH})_{2}\right]\left(\mathrm{CO}_{3}\right)_{0.14}\left(\mathrm{H}_{2} \mathrm{O}\right)_{0.66} \quad\left(\mathrm{Mg}_{2.5} \mathrm{Al}^{-\mathrm{CO}_{3}} \quad \mathrm{LDH}\right)$ (LDH-W) was synthesised using the co-precipitation method adapted from a literature procedure. ${ }^{21} \mathrm{Mg}\left(\mathrm{NO}_{3}\right)_{2} \cdot 6 \mathrm{H}_{2} \mathrm{O}$ (0.075 mol, $19.23 \mathrm{~g})$ and $\mathrm{Al}\left(\mathrm{NO}_{3}\right)_{3} \cdot 9 \mathrm{H}_{2} \mathrm{O}(0.025 \mathrm{~mol}, 9.37 \mathrm{~g})$ were dissolved in $100 \mathrm{~mL}$ deionised (DI) water to make solution A. $\mathrm{Na}_{2} \mathrm{CO}_{3}(0.05 \mathrm{~mol}, 5.30 \mathrm{~g})$ was then dissolved in another $100 \mathrm{~mL}$ DI water to make solution B. Solution A was added to solution B dropwise, while stirring, over the course of $1 \mathrm{~h}$. The mixture was kept at $\mathrm{pH} 10$ for the synthesis by the simultaneous addition of $4 \mathrm{M} \mathrm{NaOH}$ solution. When the addition of the solution A was completed and the final $\mathrm{pH}$ set to 10 , the reaction was stirred for $24 \mathrm{~h}$ at room temperature. 
After the ageing process, the LDH product was washed with DI water until pH 7 and filtered. The product was dried overnight in a vacuum oven at $30^{\circ} \mathrm{C}$.

For solvent treated samples, the wet cake was collected after the $\mathrm{LDH}$ was washed with water until the $\mathrm{pH}$ of the aqueous washing reached 7 . The $30 \%$ solid content LDH wet cake was then re-dispersed in the appropriate solvent for $4 \mathrm{~h}$ then filtered and dried. The solvents used were ethanol, acetone, isopropyl alcohol, ethyl acetate and 1-hexanol to yield LDH-E, LDH-A, LDH-IPA, LDH-EA and LDH 1H, respectively. LDH samples were pre-treated to $200{ }^{\circ} \mathrm{C}$ for $1 \mathrm{~h}$ using a temperature ramping rate of $5{ }^{\circ} \mathrm{C} \min ^{-1}$ prior to characterisation and further studies.

\section{Synthesis of ST-LDO samples}

The various ST-LDH samples were calcined in a furnace at $450{ }^{\circ} \mathrm{C}$ for $12 \mathrm{~h}$ with a ramping rate of $5{ }^{\circ} \mathrm{C} \mathrm{min}^{-1}$ and were used immediately after calcination to prevent rehydration and intercalation of impurities. The ST-LDOs were named LDO-W, LDO-E, LDO-A, LDO-IPA, LDO-EA and LDO-1H following from their parent ST-LDH respectively.

\section{Conflicts of interest}

There are no conflicts to declare.

\section{Acknowledgements}

The authors would like to thank SCG Chemicals Co., Ltd. (Thailand) and the Balliol College Mehnert Scholarship for funding (DWJL).

\section{Notes and references}

1 A. I. Khan and D. O'Hare, J. Mater. Chem., 2002, 12, 31913198.

2 W. J. L. Pesic, S. Salipurovic, V. Markovic and D. Vucelic, J. Mater. Chem., 1992, 2, 1069-1073.

3 G. Marcelin, N. J. Stockhausen, J. F. M. Post and A. Schutz, J. Phys. Chem., 1989, 93, 4646-4650.

4 M. Ogawa and K. Inomata, Chem. Lett., 2005, 34, 810-811.

5 K. L. Erickson, T. E. Bostrom and R. L. Frost, Mater. Lett., 2005, 59, 226-229.

6 F. Cavani, F. Trifirò and A. Vaccari, Catal. Today, 1991, 11, 173-301.

7 K. Chibwe and W. Jones, J. Chem. Soc., Chem. Commun., 1989, 926-927.

8 J. Rocha, M. del Arco, V. Rives and M. A. Ulibarri, J. Mater. Chem., 1999, 9, 2499-2503.

9 W. Kagunya, Z. Hassan and W. Jones, Inorg. Chem., 1996, 35, 5970-5974.

10 G. Fan, F. Li, D. G. Evans and X. Duan, Chem. Soc. Rev., 2014, 7040, 7040-7066.
11 M. B. Gawande, R. K. Pandey and R. V. Jayaram, Catal. Sci. Technol., 2012, 2, 1113.

12 S. J. Park, H. A. Ahn, I. J. Heo, I.-S. Nam, J. H. Lee, Y. K. Youn and H. J. Kim, Top. Catal., 2010, 53, 57-63.

13 J. Zhao, J. Xie, C.-T. Au and S.-F. Yin, Appl. Catal., A, 2013, 467, 33-37.

14 Q. Wang, Z. Wu, H. H. Tay, L. Chen, Y. Liu, J. Chang, Z. Zhong, J. Luo and A. Borgna, Catal. Today, 2011, 164, 198-203.

15 J. M. Silva, R. Trujillano, V. Rives, M. A. Soria and L. M. Madeira, Chem. Eng. J., 2017, 325, 25-34.

16 S. Kim, S. G. Jeon and K. B. Lee, ACS Appl. Mater. Interfaces, 2016, 8, 5763-5767.

17 H. Du, A. D. Ebner and J. A. Ritter, Ind. Eng. Chem. Res., 2011, 50, 412-418.

18 K. Coenen, F. Gallucci, G. Pio, P. Cobden, E. van Dijk, E. Hensen and M. van Sint Annaland, Chem. Eng. J., 2017, 314, 554-569.

19 N. N. A. H. Meis, J. H. Bitter and K. P. de Jong, Ind. Eng. Chem. Res., 2010, 49, 1229-1235.

20 Q. Wang and D. O'Hare, Chem. Commun., 2013, 49, 63016303.

21 C. Chen, M. Yang, Q. Wang, J.-C. Buffet and D. O'Hare, J. Mater. Chem. A, 2014, 2, 15102-15110.

22 K. Ruengkajorn, V. Erastova, J.-C. Buffet, H. C. Greenwell and D. O'Hare, Chem. Commun., 2018, 54, 4394-4397.

23 V. Erastova, M. T. Degiacomi, D. O'Hare and H. C. Greenwell, RSC Adv., 2017, 7, 5076-5083.

24 J.-C. Buffet, Z. R. Turner, R. T. Cooper and D. O'Hare, Polym. Chem., 2015, 6, 2493-2503.

25 G. E. Hickman, C. M. R. Wright, A. F. R. Kilpatrick, Z. R. Turner, J.-C. Buffet and D. O'Hare, Mol. Catal., 2019, 468, 139-147.

26 S. Shang, A. Hanif, M. Sun, Y. Tian, Y. S. Ok, I. K. M. Yu, D. C. W. Tsang, Q. Gu and J. Shang, J. Hazard. Mater., 2019, 373, 285-293.

27 X. Zhu, C. Chen, H. Suo, Q. Wang, Y. Shi, D. O'Hare and N. Cai, Energy, 2019, 167, 960-969.

28 M. Yang, O. McDermott, J.-C. Buffet and D. O'Hare, RSC Adv., 2014, 4, 51676-51682.

29 K. Ruengkajorn, C. M. R. Wright, N. H. Rees, J.-C. Buffet and D. O'Hare, Mater. Chem. Front., 2018, 2, 2277-2285.

30 K. Muramatsu, Y. Kuroda, H. Wada, A. Shimojima and K. Kuroda, Dalton Trans., 2018, 47, 3074-3083.

31 J. I. Di Cosimo, V. K. Díez, M. Xu, E. Iglesia and C. R. Apesteguía, J. Catal., 1998, 178, 499-510.

32 R. W. Stevens, R. V. Siriwardane and J. Logan, Energy Fuels, 2008, 22, 3070-3079.

33 M. J. Hudson, S. Carlino and D. C. Apperley, J. Mater. Chem., 1995, 5, 323.

34 F. Prinetto, G. Ghiotti, R. Durand and D. Tichit, J. Phys. Chem. B, 2000, 11117-11126.

35 U. Sharma, B. Tyagi and R. V. Jasra, Ind. Eng. Chem. Res., 2008, 47, 9588-9595. 